\title{
Timeliness of information disclosure during the low transmission period of COVID-19: resident-level observational study in China
}

Tingting Yang ${ }^{1 \dagger}$, Xin Shen ${ }^{2 \dagger}$, Yongguang Yang ${ }^{3}$, Yong Gan ${ }^{2}$, Jing Feng ${ }^{2}$, Zihui Lei ${ }^{2}$, Weixin Zhang ${ }^{4}$, Yuxin Zhao ${ }^{5}$ and Lijun Shen ${ }^{6 *}$

\begin{abstract}
Background: Only when people feel they have received timely disclosure will they have sufficient incentive to implement community prevention and control measures. The timely and standardized information published by authorities as a response to the crisis can better inform the public and enable better preparations for the pandemic during the low transmission period of COVID-19; however, there is limited evidence of whether people consent that information is disclosed timely and influencing factors.
\end{abstract}

Methods: A cross-sectional survey was conducted in China from 4 to 26 February 2021. Convenient sampling strategy was adopted to recruit participators. Participants were asked to filled out the questions that assessed questionnaire on the residents' attitudes to information disclosure timely. A binary logistic regression analysis was performed to identify the risk factors affecting the residents' attitudes.

Results: A total of 2361 residents filled out the questionnaire. 1704 (72.17\%) consented COVID-19 information has been disclosed timely. Furthermore, age $(\mathrm{OR}=0.093,95 \% \mathrm{Cl}=0.043 \sim 0.201)$, gender $(\mathrm{OR}=1.396$, $95 \% \mathrm{Cl}=1.085 \sim 1.797)$, place of residence $(\mathrm{OR}=0.650,95 \% \mathrm{Cl}=0.525 \sim 0.804)$, employed status $(\mathrm{OR}=2.757$, $95 \% \mathrm{Cl}=1.598 \sim 4.756)$, highest educational level $(\mathrm{OR}=0.394,95 \% \mathrm{Cl}=0.176 \sim 0.880)$, region $(\mathrm{OR}=0.561$, $95 \% \mathrm{Cl}=0.437 \sim 0.720)$ and impact on life by the COVID-19 $(\mathrm{OR}=0.482,95 \% \mathrm{Cl}=0.270 \sim 0.861)$ were mainly factors associated with residents' attitudes.

Conclusions: The aims of this study were to evaluate the residents attitudes to information disclosure timely during the low transmission period in China and to provide a scientific basis for effective information communication in future public health crises. Timely and effective efforts to disclose information need to been made during the low transmission period. Continued improvements to local authority reporting will contribute to more effective public communication and efficient public health research responses. The development of protocols and the standardization of epidemic message templates - as well as the use of uniform operating procedures to provide regular information updates — should be prioritized to ensure a coordinated national response.

\footnotetext{
*Correspondence: shenlijun2022@163.com

${ }^{\dagger}$ Tingting Yang and Xin Shen contributed equally to this work.

${ }^{6}$ Department of Clinical Research Center, Henan Provincial People's

Hospital and Zhengzhou University People's Hospital, No. 7, Weiwu Road,

Jinshui District, Zhengzhou 450003, Henan, China

Full list of author information is available at the end of the article
}

(c) The Author(s) 2022. Open Access This article is licensed under a Creative Commons Attribution 4.0 International License, which permits use, sharing, adaptation, distribution and reproduction in any medium or format, as long as you give appropriate credit to the original author(s) and the source, provide a link to the Creative Commons licence, and indicate if changes were made. The images or other third party material in this article are included in the article's Creative Commons licence, unless indicated otherwise in a credit line to the material. If material is not included in the article's Creative Commons licence and your intended use is not permitted by statutory regulation or exceeds the permitted use, you will need to obtain permission directly from the copyright holder. To view a copy of this licence, visit http://creativecommons.org/licenses/by/4.0/. The Creative Commons Public Domain Dedication waiver (http://creativecommons.org/publicdomain/zero/1.0/) applies to the data made available in this article, unless otherwise stated in a credit line to the data. 
Keywords: Information disclosure, COVID-19, Information, China, Disclosure, Low transmission period, Public attitudes, Health information

\section{Background}

In December 2019, an outbreak of COVID-19, caused by infection with SARS-CoV-2, emerged in Wuhan, Hubei Province, China, and subsequently became a global pandemic, seriously threatening the lives and health of the public and jeopardizing the stable economic development and social safety of nations $[1,2]$. In the absence of effective treatment or a vaccine, the success or failure of mitigating COVID-19 transmission in the population relies heavily on the effectiveness of social distancing, self-protection, case detection, quarantine, isolation, and testing [3]. The effectiveness of these nonpharmaceutical interventions depends on the active participation and engagement of residents in the community, which is substantially influenced by the information disclosure of COVID-19. Given the short disease transmission doubling time [4], the timing (and hence time lags) in the communication of critical public health information to the community has a profound impact on the outcome of public adherence to nonpharmaceutical measures and, ultimately, on the outcome of outbreak mitigation [5]. Only when people feel they have received timely disclosure will they have sufficient incentive to implement community prevention and control measures $[6,7]$.

Public health emergencies, especially outbreaks of new infectious diseases, are often accompanied by uncertainty about the cause of the emergency. However, the resulting information on the morbidity and mortality of the diseases becomes the focus of public concern from the moment it emerges. Simultaneously, the unknown causes of public health emergencies stimulate increased information-seeking behavior in people who are aiming to reduce their uncertainties about the emergent situation [8]. However, in the absence of information, people experience a wide range of emotions in the face of unexpected situations, and the anxiety or fear thus generated can exacerbate the occurrence [9]. In addition to the professional measures of epidemic prevention and control, keeping the information accurate and transparent are critical parts of the crisis response, reflecting the significance of the establishment of government monitoring-feedback-intervention mechanisms in public health emergencies [8]. Prompt information disclosure is a top priority for preparedness and it enables a collective response to the COVID-19 pandemic [10]. The emergence of The COVID-19 infodemic further reveals the importance of providing timely but also accurate, simple, and non-contradictory information [11, 12]. As the World Health Organization (WHO) declared the COVID-19 outbreak a public health emergency of international concern, people have the right to be clearly informed about the health risks that they and their communities face [13]. The public's feedback to the timeliness of information disclosure is an important way to reflect the government's information dissemination work. To help the general public and researchers bridge knowledge gaps and respond to the crisis in a timely manner, whether information has been disclosed timely by the China government was a crucial question.

Since the severe acute respiratory syndrome (SARS) emergency in 2003 and the avian influenza A (H7N9) epidemic in 2013, the Chinese government's provision of rapid, effective, and efficient disclosure of epidemic information has substantially improved [13, 14]. Starting on January 3, 2020, information regarding COVID19 cases has been reported to the WHO and the general public through China's National Health Commission on a daily basis [15]. The time interval from the first case description to the identification of the pathogen on January 7-as well as the availability of probes for PCR detection on January 21-was much faster for COVID19 than for SARS [16]. The National Health Commission took prompt public health measures and soon classified COVID-19 as a new notifiable disease under the National Infectious Disease Law and the Frontier Health and Quarantine Law on January 20, 2020, thus authorizing by law the prompt disclosure of information about the epidemic at the subnational level [15]. During the epidemic, people expressed high satisfaction with the timeliness of information disclosure $[17,18]$.

Disclosing information timely and having communitybased approaches have been shown to play a significant role in addressing the issues brought about by the COVID-19 outbreak in China. Previous global studies have confirmed the residents' concern about the epidemic information [19], and some studies have examined the impact of information disclosure on authenticity screening [20]. Some studies have focused on channels [21] and countermeasures [22], but there is no research to observe the timeliness of information disclosure from the perspective of residents. Currently COVID-19 epidemic has been controlled and China has entered a period of low transmission [23]. At this stage, China government still need to disclose information timely. Whether the residents consent they have timely access to COVID19 information is worth to survey in China. As more 
and more countries enter the low-transmission phase, maintaining prevention awareness among the disclosing information timely is critical to preventing a secondary outbreak. By investigating the whether people consent that information is disclosed timely and influencing factors of different people's attitudes towards disclosure in China, we can reflect the prevention awareness of the government and residents during the low transmission period of COVID-19, which can provide a reference for Chinese and global public health policy makers.

\section{Methods}

\section{Ethics statement}

This study protocol was approved by the institutional review board of Tongji Medical College of Huazhong University of Science and Technology, Wuhan, China. All methods were performed in accordance with the relevant guidelines and regulations. Respondents were informed that their participation was voluntary, and consent was implied on the completion of the questionnaire.

\section{Study participants and survey design}

A cross-sectional survey was conducted in China from 4 to 26 February 2021 . We stratify the respondents mainly according to the eastern, central and western region of China. We selected residents from eastern (Beijing, Tianjin, Hebei, Liaoning, Shanghai, Jiangsu, Zhejiang, Fujian, Shandong, Guangdong and Hainan), central (Shanxi, Jilin, Heilongjiang, Anhui, Jiangxi, Henan, Hubei, and Hunan) and western (Chongqing, Sichuan, Guizhou, Yunnan, Tibet, Shaanxi, Gansu, Qinghai, Ningxia, Xinjiang, Inner Mongolia, and Guangxi) China to complete the survey. Convenient sampling strategy was adopted to recruit participators; the research team used WeChat (the most popular social media platform in China) to advertise and circulate the survey link to their network members. Network members were requested to distribute the survey invitation to all their contacts. The participants were informed that their participation was voluntary, and consent was implied through their completion of the questionnaire. The inclusion criteria were that the respondents were Chinese citizens who were at least 18 years old, and able to comprehend and read Chinese.

\section{Instruments}

The questionnaire consisted of two parts: 1) socio-demographic characteristics, with 8 items, including gender, age, marital status, highest educational level, place of residence, religion, employment status and have a chronic disease (diagnosed by a doctor); and 2) the recognition to COVID-19 information disclosing timely, with 3 items, including "consent COVID-19 information has been disclosed timely", "the primary way to get information about the COVID-19" and "COVID-19 have a large impact on your life". (Supplement questionnaire format).

Wenjuanxing (www.wjx.cn), a widely used platform for conducting surveys in China, was used to develop the electronic questionnaire. An online poster with an access code or the website link to the questionnaire was distributed via two ways: (1) posted on our WeChat; and (2) distributed via WeChat groups, with an average of one to two RMB each as compensation. Each individual could only participate once on each WeChat account to avoid repeated submissions.

\section{Statistical methods}

The data were analysed using SPSS ${ }^{\mathrm{TM}}$ for Windows, Version 22.0 (SPSS, Inc., Chicago, IL, USA). We dichotomous the answers to the "Consent COVID-19 information has been disclosed timely" of residents' attitudes as "Yes" and "No". The descriptive statistics were presented as the number of observations with percentage (\%), and we analyzed the difference in demographic statistics by Chisquare $(x 2)$ test. Shapiro-Wilk test is used to judge the normality of quantitative data. Due to the disparities in socioeconomic status in different regions, the data have a typical hierarchical structure. The verification of specific assumptions of the logistic regressions (e.g., low multicollinearity, independence of errors, linearity in the logit for continuous variables, and lack of strongly influential outliers) were tested [24]. We performed a mixed-effect logistic regression model with a random cluster effect (geographic regions) to investigate the adjusted OR (95\% CI) of influencing factors of residents' attitudes to agree they acquire adequate community protection. Further, we explored the factors influencing participants' attitudes in Eastern, Central, and Western China, respectively, through multivariable logistic regression analysis. $P$-values are more suitable as a measure of the strength of evidence against the null hypothesis [25]. Therefore, we talked about degrees of evidence instead of significance and non-significance (e.g., $P=.049$ and $P=.051$ are similar results). In the classification of evidence in our study, we divided it into two types: "high" and "low".

\section{Results}

\section{Descriptive statistics}

A total of 2453 residents received the questionnaire, of which 21 participants did not respond and 71 questionnaires were not filled. The response rate was $96.24 \%$ and 2361 complete questionnaires were employed for results analysis. Table 1 reports the social-demographic characteristics of 2361 respondents. The median age was 34 years (Interquartile range $=26-42$ ) and majority of respondents were female (60.10\%). Among the 
Table 1 Statistical description of study samples: univariate Analysis of the differences of residents' attitudes to consent COVID-19 information has been disclosed timely during the low transmission period

\begin{tabular}{|c|c|c|c|c|}
\hline Variables & $\mathrm{N}(\%)$ & $x^{2}$ & $P$ & $\begin{array}{l}\text { Evidence } \\
\text { Degrees }\end{array}$ \\
\hline Total & $2361(100)$ & NA & NA & NA \\
\hline \multicolumn{5}{|c|}{ Consent COVID-19 information has been disclosed timely } \\
\hline Yes & $1704(72.17)$ & NA & NA & NA \\
\hline No & $657(27.83)$ & & & \\
\hline \multicolumn{5}{|l|}{ Gender } \\
\hline Male & $942(39.90)$ & 0.415 & 0.520 & Low \\
\hline Female & $1419(61.10)$ & & & \\
\hline \multicolumn{5}{|l|}{ Age group, y } \\
\hline $18-44$ & $1845(78.14)$ & 64.094 & $<0.001$ & High \\
\hline $45-59$ & $369(15.63)$ & & & \\
\hline$>60$ & $111(4.70)$ & & & \\
\hline \multicolumn{5}{|l|}{ Highest educational level } \\
\hline Primary school or below & $68(2.88)$ & 13.475 & 0.001 & High \\
\hline Middle school & $186(7.88)$ & & & \\
\hline College degree or above & $2107(89.24)$ & & & \\
\hline \multicolumn{5}{|l|}{ Place of residence } \\
\hline Urban & $1372(58.11)$ & 5.762 & 0.016 & High \\
\hline Rural & 989 (41.89) & & & \\
\hline \multicolumn{5}{|l|}{ Region } \\
\hline Eastern China & $421(17.83)$ & 35.886 & $<0.001$ & High \\
\hline Central China & $1470(62.26)$ & & & \\
\hline Western China & $470(19.91)$ & & & \\
\hline \multicolumn{5}{|l|}{ Employment status } \\
\hline Employed & $1014(42.95)$ & 8.721 & 0.003 & High \\
\hline Unemployed & $1347(57.05)$ & & & \\
\hline \multicolumn{5}{|c|}{ Have a chronic disease (diagnosed by a doctor) } \\
\hline Yes & $1621(68.66)$ & 0.093 & 0.761 & Low \\
\hline No & $740(31.34)$ & & & \\
\hline \multicolumn{5}{|l|}{ Marital status } \\
\hline Unmarried & $1560(66.07)$ & 5.929 & 0.015 & High \\
\hline Married & $801(33.93)$ & & & \\
\hline \multicolumn{5}{|c|}{ The primary way to get information about the COVID-19 } \\
\hline $\begin{array}{l}\text { Traditional Media (Televi- } \\
\text { sion, Radio and Newspa- } \\
\text { pers) }\end{array}$ & $1361(57.65)$ & 0.001 & 0.980 & Low \\
\hline $\begin{array}{l}\text { Emerging media (Weibo, } \\
\text { WeChat and Interent news) }\end{array}$ & $1000(42.35)$ & & & \\
\hline \multicolumn{5}{|c|}{ COVID-19 have a large impact on your life } \\
\hline Strongly disagree & $620(26.26)$ & 7.825 & 0.098 & Low \\
\hline Disagree & $964(40.83)$ & & & \\
\hline Not sure & $562(23.80)$ & & & \\
\hline Agree & $124(5.25)$ & & & \\
\hline Strongly agree & $91(3.85)$ & & & \\
\hline
\end{tabular}

respondents, 421 (17.83\%), 1470 (62.26\%), and 470 (19.91\%) were from eastern, central, and western China, respectively. Most respondents (89.24\%) have the bachelor's degree or higher. More than half of the participants (57.05\%) were unemployed.

Of them, 1704 (72.17\%) consented COVID-19 information has been disclosed timely. The Variance Inflation Factor (VIF) value among the variables was less than five, indicating that there is low multicollinearity. Durbin-Waston test shows that the DW value is 1.67 (close to 2), which verifies the independence of errors. Since all variables are categorical in this study, linearity in the logit for continuous variables is not considered. In addition, significant outliers are eliminated by $\mathrm{k}$-means algorithm. Results of the analysis suggested the gender, age, place of residence, region, employment status and marital status were high statistical significance influencing factors for "consenting COVID-19 information has been disclosed timely" (Table 1). Considering the significant differences different geographic regions in the sampling, we conducted further analyses with participants from Eastern, Central, and Western China, respectively (Table 2). In the mixed-effect logistic regression analysis, Chinese residents who were older $(\mathrm{OR}=0.093,95 \% \mathrm{CI}=0.043 \sim 0.201)$, lived in urban $(\mathrm{OR}=0.650,95 \% \mathrm{CI}=0.525 \sim 0.804)$, lived in central China ( $\mathrm{OR}=0.561,95 \% \mathrm{CI}=0.437 \sim 0.720)$, and strongly agreed that COVID-19 have a large impact on your life $(\mathrm{OR}=0.482,95 \% \mathrm{CI}=0.270 \sim 0.861)$ were not more likely to consent COVID-19 information has been disclosed timely (Table 3).

In addition, we stratified the study sample by regions and conducted multivariate logistic regression analyses. Male $(\mathrm{OR}=1.396,95 \% \mathrm{CI}=1.085 \sim 1.797)$ and employed $(\mathrm{OR}=2.757,95 \% \mathrm{CI}=1.598 \sim 4.756)$ were also the factors associated with an increased willingness to consent COVID-19 information has been disclosed timely in Central China and Western China respectively; while the highest educational level is college degree or above $(\mathrm{OR}=0.394,95 \% \mathrm{CI}=0.176 \sim 0.880)$ was a deduced factor in Central China (Table 4).

\section{Discussion}

Our study is based on a cross-sectional survey, determined the residents' attitudes to consent COVID-19 information has been disclosed timely and influencing factors. We found that 1704 (72.17) of residents consent COVID-19 information has been disclosed timely during the low transmission period of the COVID-19. Furthermore, age, gender, place of residence, employed status, highest educational level, region and impact on life by the COVID-19 were mainly factors associated with residents' attitudes. 


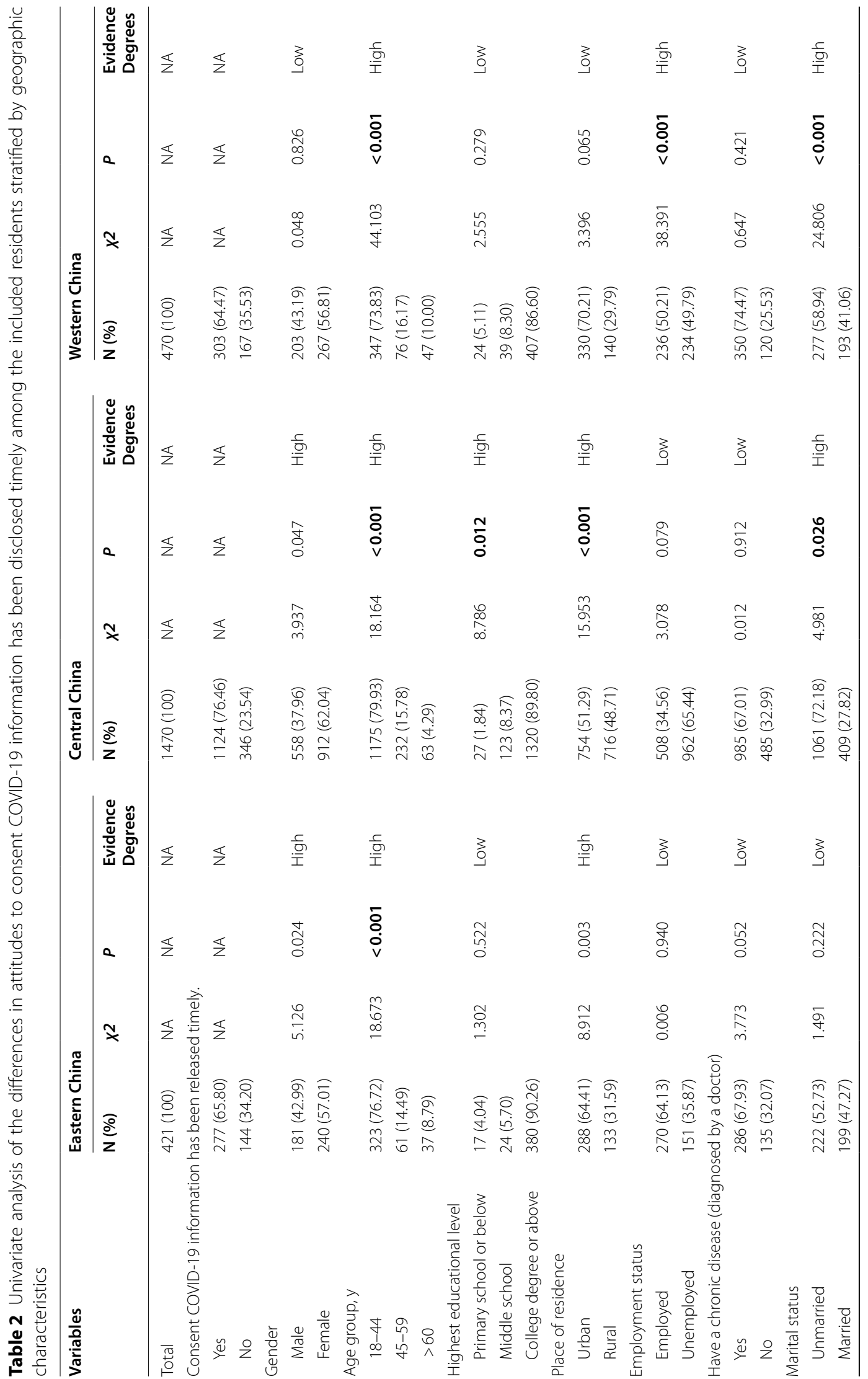




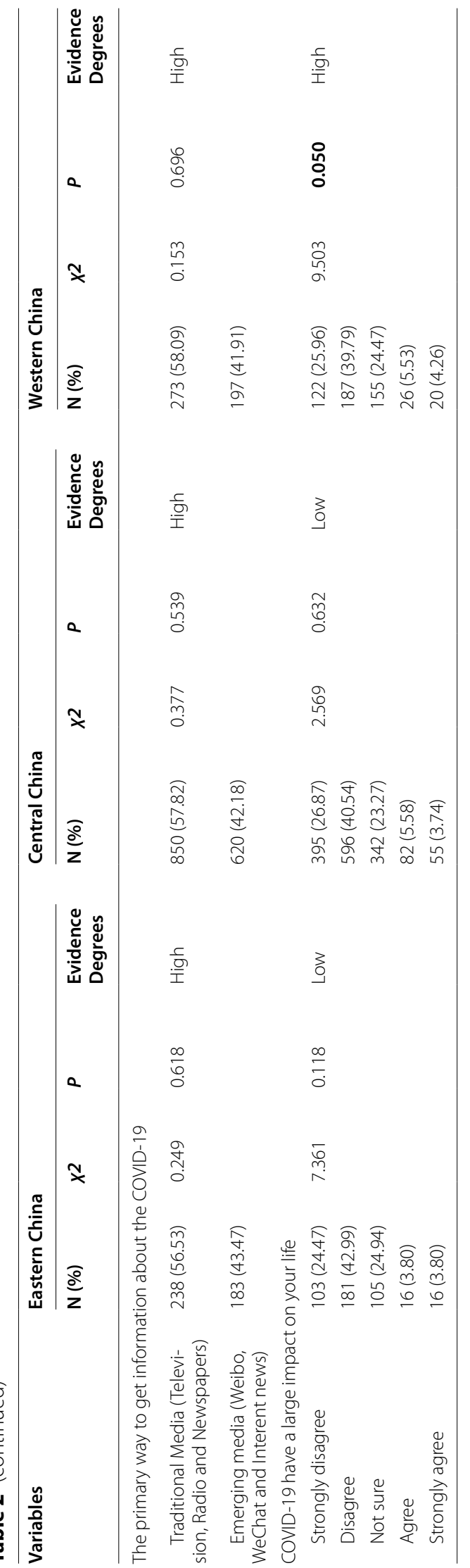


Table 3 Mixed-Effect Logistic Regression Analysis on the Influencing Factors of Residents' attitudes to consent COVID-19 information has been disclosed timely during the low transmission period of the COVID-19

\begin{tabular}{|c|c|c|c|c|c|c|}
\hline Variables & Coefficient & S.E. & $P$ & Evidence Degrees & OR & $95 \% \mathrm{Cl}$ \\
\hline \multicolumn{7}{|l|}{ Gender (Ref: Female) } \\
\hline Male & 0.109 & 0.097 & 0.262 & Low & 1.115 & $0.922 \sim 1.350$ \\
\hline \multicolumn{7}{|l|}{ Age group, y (Ref: 18-44) } \\
\hline $45-59$ & -0.648 & 0.144 & $<0.001$ & High & 0.523 & $0.394 \sim 0.694$ \\
\hline$>60$ & -2.378 & 0.394 & $<0.001$ & & 0.093 & $0.043 \sim 0.201$ \\
\hline \multicolumn{7}{|c|}{ Highest educational level (Ref: Primary school or below) } \\
\hline Middle school & -0.202 & 0.306 & 0.509 & Low & 0.817 & $0.448 \sim 1.489$ \\
\hline College degree or above & -0.403 & 0.266 & 0.129 & Low & 0.668 & $0.397 \sim 1.125$ \\
\hline \multicolumn{7}{|l|}{ Place of residence (Ref: Rural) } \\
\hline Urban & -0.431 & 0.109 & $<0.001$ & High & 0.650 & $0.525 \sim 0.804$ \\
\hline \multicolumn{7}{|l|}{ Region (Ref: Eastern China) } \\
\hline Central China & -0.578 & 0.128 & $<0.001$ & High & 0.561 & $0.437 \sim 0.720$ \\
\hline Western China & 0.132 & 0.147 & 0.370 & Low & 1.141 & $0.855 \sim 1.523$ \\
\hline \multicolumn{7}{|l|}{ Employment status (Ref: Unemployed) } \\
\hline Employed & 0.232 & 0.134 & 0.083 & Low & 1.261 & $0.970 \sim 1.639$ \\
\hline \multicolumn{7}{|c|}{ Have a chronic disease (diagnosed by a doctor) (Ref: No) } \\
\hline Yes & -0.084 & 0.103 & 0.414 & Low & 0.92 & $0.752 \sim 1.124$ \\
\hline \multicolumn{7}{|l|}{ Marital status (Ref: Unmarried) } \\
\hline Married & 0.12 & 0.135 & 0.375 & Low & 1.128 & $0.865 \sim 1.471$ \\
\hline \multicolumn{7}{|c|}{ The primary way to get information about the COVID-19 [Ref:Traditional Media (Television, Radio and Newspapers)] } \\
\hline $\begin{array}{l}\text { Emerging media (Weibo, WeChat } \\
\text { and Interent news) }\end{array}$ & 0.008 & 0.096 & 0.932 & Low & 1.008 & $0.835 \sim 1.217$ \\
\hline \multicolumn{7}{|c|}{ COVID-19 have a large impact on your life (Ref: Strongly disagree) } \\
\hline Disagree & -0.164 & 0.117 & 0.162 & Low & 0.849 & $0.674 \sim 1.068$ \\
\hline Not sure & -0.125 & 0.133 & 0.347 & Low & 0.883 & $0.681 \sim 1.145$ \\
\hline Agree & -0.209 & 0.239 & 0.381 & Low & 0.811 & $0.508 \sim 1.296$ \\
\hline Strongly agree & -0.729 & 0.296 & 0.014 & High & 0.482 & $0.270 \sim 0.861$ \\
\hline
\end{tabular}

COVID-19 disproportionately impacts vulnerable populations [26]. Access to health information has long been limited for many people, and access barriers and health disparities are likely exacerbated during this pandemic [27]. Older, female, unemployed and those impacted by COVID-19 expressed concern about the lack of timely information disclosing in this study. In fact, these groups were vulnerable in the COVID-19 pandemic and therefore need more adequate information support, which suggested that the government should pay more attention to ensuring the information of vulnerable groups in the process of information disclosure. On the other hand, residents who live in urban and have college degree or above showed higher expectations for information disclosure. A possibility is that these people are more aware of the importance of disclosure, and therefore more eager to get full information. This finding also provides further evidence for the relationship between cognitive level and health needs.
Varieties of information dissemination channels make it easier for people to obtain the information. In addition to the traditional way of disseminating information by word of mouth, we can also get information from online social networks, news websites and television stations, to name only a few [28]. Another finding of our study is that access to information does not affect residents' perceptions of the timeliness of information disclosure. This may be due to the emergence of multiple types of media, people are getting used to getting information from multiple sources and placing the same importance on these information. The epidemic spreading can trigger epidemic-relevant information to disseminate among the population [29] and the government should use a variety of channels to disclose information, promote the popularization of information.

When a new infectious disease breaks out, residents, especially where the epidemic is more severe, may bear heavier psychological stress [30,31]. Some studies 
Table 4 Stepwise Multivariate Logistic Regression Analysis on the Influencing Factors of Residents' attitudes to consent COVID-19 information has been disclosed timely during the low transmission period of the COVID-19

\begin{tabular}{|c|c|c|c|c|c|c|}
\hline Variables & Coefficient & S.E. & $P$ & Evidence Degrees & OR & $95 \% \mathrm{Cl}$ \\
\hline \multicolumn{7}{|l|}{ Eastern China } \\
\hline \multicolumn{7}{|l|}{ Age group, y (Ref: 18-44) } \\
\hline$>60$ & -2.336 & 0.75 & 0.002 & High & 0.097 & $0.022 \sim 0.420$ \\
\hline \multicolumn{7}{|l|}{ Place of residence (Ref: Rural) } \\
\hline Urban & -0.698 & 0.259 & 0.007 & High & 0.498 & $0.299 \sim 0.828$ \\
\hline \multicolumn{7}{|l|}{ Central China } \\
\hline \multicolumn{7}{|l|}{ Gender (Ref: Female) } \\
\hline Male & 0.334 & 0.129 & 0.009 & High & 1.396 & $1.085 \sim 1.797$ \\
\hline \multicolumn{7}{|l|}{ Age group, y (Ref: 18-44) } \\
\hline $45-59$ & -0.416 & 0.185 & 0.025 & High & 0.660 & $0.459 \sim 0.948$ \\
\hline$>60$ & -1.826 & 0.600 & 0.002 & High & 0.161 & $0.050 \sim 0.522$ \\
\hline \multicolumn{7}{|c|}{ Highest educational level (Ref: Primary school or below) } \\
\hline College degree or above & -0.932 & 0.41 & 0.023 & High & 0.394 & $0.176 \sim 0.880$ \\
\hline \multicolumn{7}{|l|}{ Place of residence (Ref: Rural) } \\
\hline Urban & -0.431 & 0.14 & 0.002 & High & 0.65 & $0.494 \sim 0.856$ \\
\hline \multicolumn{7}{|l|}{ Western China } \\
\hline \multicolumn{7}{|l|}{ Age group, y (Ref: 18-44) } \\
\hline $45-59$ & -1.155 & 0.348 & 0.001 & High & 0.315 & $0.159 \sim 0.623$ \\
\hline$>60$ & -2.722 & 0.749 & $<0.001$ & High & 0.066 & $0.015 \sim 0.286$ \\
\hline \multicolumn{7}{|c|}{ Employment status (Ref: Unemployed) } \\
\hline Employed & 1.014 & 0.278 & $<0.001$ & High & 2.757 & $1.598 \sim 4.756$ \\
\hline \multicolumn{7}{|c|}{ COVID-19 have a large impact on your life (Ref: Strongly disagree) } \\
\hline Strongly agree & -2.157 & 1.068 & 0.043 & High & 0.116 & $0.014 \sim 0.939$ \\
\hline
\end{tabular}

showed that psychological health problem among residents in Wuhan, where the epidemic was most severe, were higher than elsewhere,and were strongly correlated with the severity of the epidemic and closely mirrored the number of new cases per day [32, 33]. Our study reported that residents in central China expressed more concern about the timeliness of information disclosure. One possible reason is that COVID-19 outbreaks have mainly occurred in central China, especially in Hubei province, where residents have a greater demand for information disclosure. This further illustrates the profound impact of the pandemic on society, namely, the increased demand for government information disclosure and, based on this, the awakening of higher expectations for public health and government services.

\section{Strengths and limitations}

This study is the first study reported residents' attitudes to consent COVID-19 information has been disclosed timely and influencing factors, which can reflect awareness of prevention COVID-19 during the low transmission period. We used a nationwide sample of the Chinese population. This study provided a scientific basis for effective information communication in future public health crises, which are of importance to China and other countries.

However, this study has some limitations. First, this study used social media as the main method to disseminate the survey. Participants without access to the internet were probably not included. Second, the distribution of the study participants was imbalanced across regions (421: 1470: 470); therefore, the subgroups of variables might not be representative of the population. Third, this study could not determine how many participants reviewed the online poster or survey but decided not to complete the survey; thus, the presence of nonresponse bias could not be assessed. Finally, as the behaviors were self-reported, reporting bias was possible. This study is based on a cross-sectional survey and cannot prove a clear causal relationship. In addition, it should also be noted that the intention to stay informed does not necessarily correspond to the predisposition to take action. Indeed, phenomena such as pandemic fatigue reduce compliance with anti-pandemic norms [34]. Overall, generalization of the results should be regarded with caution. 


\section{Conclusions}

Promoting the timely disclosure of information related to public health emergencies and providing the public with regular channels through which authoritative up-to-date information is disclosed are both essential for the timely communication of risk information and guidance. This study reported that 1704 (72.17) of residents consent COVID-19 information has been disclosed timely during the low transmission period of the COVID-19. Furthermore, residents who were female, older, unemployed, lived in urban and central China, impacted by COVID19, and had college degree or above expressed stronger concerns about the timeliness of information disclosure.

Our results augment the awareness of information and data disclosed timely during the low transmission period of the COVID-19 in China. Therefore, the development of uniform protocols and standards of epidemic message templates must be encouraged, as should the use of standard operating procedures to regularly update all vital information in a manner that the public can easily interpret and researchers can effectively analyze. As more and more countries enter the low-transmission phase, this study suggest that these issues should be considered a critical policy priority for the national health authorities in China and most countries worldwide.

\section{Supplementary Information}

The online version contains supplementary material available at https://doi. org/10.1186/s12889-022-12804-x.

\section{Additional file 1.}

\section{Acknowledgments}

We thank all the participants in this study.

\section{Authors' contributions}

T.Y., X.S. and L.S. conceived and designed the study. Y.Y., J.F. and Z.L. participated in the acquisition of data. T.Y. and X.S. analyzed the data. W.Z. and Y.Z gave advice on methodology. T.Y. and X.S. drafted the manuscript. Y.G. and S.L. revised the manuscript. All authors read and approved the final manuscript. S.L. is the guarantor of this work and had full access to all the data in the study and takes responsibility for its integrity and the accuracy of the data analysis.

\section{Funding}

This study was supported by the Natural Science Foundation of Henan Province (202300410411) and the Fundamental Research Funds for the Central Universities (2020kfyXJJS059). The fund provided the money to carry out the investigation. The fund provider did not participate in the specific work of the study or undertake the specific task.

\section{Availability of data and materials}

Data may be made available by contacting the corresponding author.

\section{Declarations}

\section{Ethics approval and consent to participate}

All individuals provided written informed consent. This study was performed in line with the principles of the Declaration of Helsinki. This study protocol was approved by the institutional review board of Tongji Medical College of
Huazhong University of Science and Technology, Wuhan, China. All methods were carried out in accordance with the Chinese Statistical Law to ensure that participants' personal information was kept confidential. All experimental protocols were approved by the institutional review board of Tongji Medical College of Huazhong University of Science and Technology, Wuhan, China.

\section{Consent for publication}

Not applicable.

\section{Competing interests}

We declare that we have no conflict of interests.

\section{Author details}

${ }^{1}$ Department of Nutrition, Henan Provincial People's Hospital and Zhengzhou University People's Hospital, Zhengzhou, Henan, China. ${ }^{2}$ Department of Social Medicine and Health Management, School of Public Health, Tongji Medical College, Huazhong University of Science and Technology, Wuhan, Hubei, China. ${ }^{3}$ Department of Research Management, Henan Provincial People's Hospital and Zhengzhou University People's Hospital, Zhengzhou, Henan, China. ${ }^{4}$ School of Public Health, Jilin University, Changchun, Jilin, China. ${ }^{5}$ Community Health Service Management Center, Shenzhen Fuyong People's Hospital, Shenzhen, Guangdong, China. ${ }^{6}$ Department of Clinical Research Center, Henan Provincial People's Hospital and Zhengzhou University People's Hospital, No. 7, Weiwu Road, Jinshui District, Zhengzhou 450003, Henan, China.

Received: 21 August 2021 Accepted: 17 February 2022

Published online: 01 March 2022

\section{References}

1. Hui DS, AE I, Madani TA, Ntoumi F, Kock R, Dar O, et al. The continuing 2019-nCoV epidemic threat of novel coronaviruses to global health - The latest 2019 novel coronavirus outbreak in Wuhan, China. Int J Infect Dis. 2020;91:264-6.

2. Chen N, Zhou M, Dong X, Qu J, Gong F, Han Y, et al. Epidemiological and clinical characteristics of 99 cases of 2019 novel coronavirus pneumonia in Wuhan, China: a descriptive study. Lancet. 2020;395(10223):507-13.

3. Askitas N, Tatsiramos K, Verheyden B. Estimating worldwide effects of non-pharmaceutical interventions on COVID-19 incidence and population mobility patterns using a multiple-event study. Sci Rep-UK. 2021:11(1):1972.

4. Pellis L, Scarabel F, Stage HB, Overton CE, Chappell L, Fearon E, et al. Challenges in control of COVID-19: short doubling time and long delay to effect of interventions. Philos Trans R Soc Lond B Biol Sci. 2021;376(1829):20200264.

5. Moon S, Sridhar D, Pate MA, Jha AK, Clinton C, Delaunay S, et al. Will Ebola change the game? Ten essential reforms before the next pandemic. The report of the Harvard-LSHTM Independent Panel on the Global Response to Ebola. Lancet. 2015;386(10009):2204-21.

6. Xia H, An W, Li J, Zhang ZJ. Outlier knowledge management for extreme public health events: Understanding public opinions about COVID-19 based on microblog data. Socio-Econ Plan Sci. 2020:100941.

7. Li J, Xu Q, Cuomo R, Purushothaman V, Mackey T. Data Mining and Content Analysis of the Chinese Social Media Platform Weibo During the Early COVID-19 Outbreak: Retrospective Observational Infoveillance Study. JMIR Public Health Surveill. 2020;6(2):e18700.

8. Chen B, Chen X, Pan J, Liu K, Xie B, Wang W, et al. Dissemination and Refutation of Rumors During the COVID-19 Outbreak in China: Infodemiology Study. J Med Internet Res. 2021;23(2):e22427.

9. Zeng R, Zhu D. A model and simulation of the emotional contagion of netizens in the process of rumor refutation. Sci Rep. 2019;9(1):14164.

10. Wang C, Horby PW, Hayden FG, Gao GF. A novel coronavirus outbreak of global health concern. Lancet. 2020:395(10223):470-3.

11. WHO. Managing the covid 19 infodemic promoting healthy behaviours and mitigating the harm from misinformation and disinformation. 2020.

12. WHO. The Covid-19 infodemic. 2021.

13. Wong J, Wai A, Zhao S, Yip F, Lee JJ, Wong C, et al. Association of Individual Health Literacy with Preventive Behaviours and Family Well-Being 
during COVID-19 Pandemic: Mediating Role of Family Information Sharing. Int J Environ Res Public Health. 2020;17(23):8838.

14. Wang Y. The H7N9 influenza virus in China--changes since SARS. N Engl J Med. 2013;368(25):2348-9.

15. Hu G, Li P, Yuan C, Tao C, Wen H, Liu Q, et al. Information Disclosure During the COVID-19 Epidemic in China: City-Level Observational Study. J Med Internet Res. 2020;22(8):e19572.

16. Wilder-Smith A, Chiew CJ, Lee VJ. Can we contain the COVID-19 outbreak with the same measures as for SARS? Lancet Infect Dis. 2020;20(5):e102-7.

17. Yin F, Xia X, Song N, Zhu L, Wu J. Quantify the role of superspreaders -opinion leaders- on COVID-19 information propagation in the Chinese Sina-microblog. PLoS One. 2020;15(6):e234023.

18. Wang J, Zhou Y, Zhang W, Evans R, Zhu C. Concerns Expressed by Chinese Social Media Users During the COVID-19 Pandemic: Content Analysis of Sina Weibo Microblogging Data. J Med Internet Res. 2020;22(11):e22152.

19. Zhao Y, Cheng S, Yu X, Xu H. Chinese Public's Attention to the COVID-19 Epidemic on Social Media: Observational Descriptive Study. J Med Internet Res. 2020;22(5):e18825.

20. Huang X, Li G, Wang Y, Li X. Research on the Influence Mechanism of Epidemic Information Disclosure on Screening Authenticity Information. Procedia Comput Sci. 2021;187:109-15.

21. Liu Q, Zheng Z, Zheng J, Chen Q, Liu G, Chen S, et al. Health Communication Through News Media During the Early Stage of the COVID-19 Outbreak in China: Digital Topic Modeling Approach. J Med Internet Res. 2020;22(4):e19118.

22. Pian W, Chi J, Ma F. The causes, impacts and countermeasures of COVID19 "Infodemic": A systematic review using narrative synthesis. Inform Process Manag. 2021;58(6):102713.

23. Izda V, Jeffries MA, Sawalha AH. COVID-19: A review of therapeutic strategies and vaccine candidates. Clin Immunol. 2021:222:108634

24. Stoltzfus JC. Logistic Regression: A Brief Primer. Acad Emerg Med. 2011;18(10):1099-104.

25. Amrhein $V$, Korner-Nievergelt $F$, Roth $T$. The earth is flat ( $p>0.05)$ : significance thresholds and the crisis of unreplicable research. PEERJ. 2017; 5:e3544

26. Egede $L E$, Ruggiero KJ, Frueh $B C$. Ensuring mental health access for vulnerable populations in COVID era. J Psychiatr Res. 2020;129:147-8.

27. Kuy S, Tsai R, Bhatt J, Chu QD, Gandhi P, Gupta R, et al. Focusing on Vulnerable Populations During COVID-19. Acad Med. 2020;95(11):e2-3.

28. Wang Z, Xia C. Co-evolution spreading of multiple information and epidemics on two-layered networks under the influence of mass media. Nonlinear Dynam. 2020:1-14.

29. Wu Q, Fu X, Small M, Xu XJ. The impact of awareness on epidemic spreading in networks. Chaos. 2012;22(1):13101.

30. Cao H, Zuo C, Li G, Huang Y, Li L, Huang S, et al. A Cross-Sectional Study of Psychological Status in Different Epidemic Areas in China After the COVID-19 Outbreak. Front Psychiatry. 2020;11:575705.

31. Leung GM, Ho LM, Chan SK, Ho SY, Bacon-Shone J, Choy RY, et al. Longitudinal assessment of community psychobehavioral responses during and after the 2003 outbreak of severe acute respiratory syndrome in Hong Kong. Clin Infect Dis. 2005;40(12):1713-20.

32. Xiaoming $X$, Ming A, Su H, Wo W, Jianmei C, Qi Z, et al. The psychological status of 8817 hospital workers during COVID-19 Epidemic: A crosssectional study in Chongqing. J Affect Disord. 2020;276:555-61.

33. Liu Y, Chen H, Zhang N, Wang X, Fan Q, Zhang Y, et al. Anxiety and depression symptoms of medical staff under COVID-19 epidemic in China. J Affect Disord. 2021;278:144-8

34. WHO. Pandemic fatigue: reinvigorating the public to prevent COVID-19: policy considerations for Member States in the WHO European Region. 2020.

\section{Publisher's Note}

Springer Nature remains neutral with regard to jurisdictional claims in published maps and institutional affiliations.
Ready to submit your research? Choose BMC and benefit from:

- fast, convenient online submission

- thorough peer review by experienced researchers in your field

- rapid publication on acceptance

- support for research data, including large and complex data types

- gold Open Access which fosters wider collaboration and increased citations

- maximum visibility for your research: over $100 \mathrm{M}$ website views per year

At BMC, research is always in progress.

Learn more biomedcentral.com/submissions 